\title{
Insomnia in patients on hemodialysis for a short versus long duration
}

\author{
This article was published in the following Dove Press journal: \\ Neuropsychiatric Disease and Treatment \\ 2 September 2016 \\ Number of times this article has been viewed
}

\author{
Tetsu Tomita' \\ Norio Yasui-Furukori' \\ Masaki Oka' \\ Takaaki Shimizu² \\ Aya Nagashima ${ }^{2}$ \\ Kento Mitsuhashi ${ }^{2}$ \\ Hisao Saito ${ }^{3}$ \\ Kazuhiko Nakamura' \\ 'Department of Neuropsychiatry, \\ Graduate School of Medicine, \\ ${ }^{2}$ School of Medicine, Hirosaki \\ University, ${ }^{3}$ Department of Urology, \\ Oyokyo Kidney Research Institute, \\ Hirosaki, Japan
}

Background: Many studies have investigated insomnia and the factors associated with this condition in hemodialysis (HD) patients, although the influence of HD duration has not been thoroughly investigated. In the present study, we investigated the factors, especially the duration of HD, associated with insomnia in HD patients.

Patients and methods: A total of 138 patients undergoing HD were recruited, and the Japanese version of the Pittsburgh Sleep Quality Index (PSQI) was used to assess the quality of sleep. Subjects with a total PSQI score up to 4 and those with a score of at least 5 were identified as normal subjects and subjects with insomnia, respectively. Additionally, we assessed restless legs syndrome, depression using the Center for Epidemiologic Studies Depression Scale, and health-related quality of life (QOL) using the Short Form 8 Health Survey. We divided the subjects into two groups according to the median HD duration.

Results: The prevalence rate of insomnia was $54.3 \%$ among all the subjects. Twenty-one subjects (15.2\%) had depression, 26 (18.8\%) had restless legs syndrome, and $75(54.3 \%)$ had insomnia. The median HD duration was 4 years. The scores of components 1 and 4 of the PSQI, subjective sleep quality and habitual sleep efficiency, did not show a significant difference between the normal and insomnia groups. The score of component 7 , daytime dysfunction, showed a significant difference between the short and long HD duration groups. In multiple regression analysis, the score of the Short Form 8 Health Survey showed a significant association with the PSQI score in the long HD duration group, but no variable showed a significant association in the short HD duration group.

Conclusion: Patients with a longer duration of HD indicated that insomnia has an influence on their daily activities, with a significant association between insomnia and QOL. Greater attention should be paid to poor QOL and troubles in daily activities caused by insomnia in patients with a longer HD duration.

Keywords: sleep disorders, kidney, quality of life, duration of hemodialysis

\section{Introduction}

Patients with chronic disease frequently have comorbid psychiatric disease or psychological distress. Many studies have investigated the association between end-stage renal disease or hemodialysis (HD) and depression. ${ }^{1-7}$ A review of the literature shows that $25 \%-50 \%$ of HD patients exhibit depressive symptoms. ${ }^{1,2,8}$ Although some single-center studies reported rates of depression or major depressive disorder in HD patients of $25 \%-30 \%,{ }^{9-11}$ a multicenter international study showed large differences in rates between the countries where the investigations were conducted; for instance, the rate was $2 \%$ in Japan but $21.7 \%$ in the US. ${ }^{12}$

Insomnia is one of the symptoms of depression, and many recent studies have reported an association between insomnia and HD. ${ }^{13-17}$ Almost all of these reports
Correspondence: Tetsu Tomita Department of Neuropsychiatry, Graduate School of Medicine, Hirosaki University, 5 Zaifu-cho, Hirosaki 036-8562, Japan

Tel +8I I72395066

Fax $+81 \quad 172395067$

Email ttomita1984@yahoo.co.jp 
showed a higher prevalence of insomnia or restless legs syndrome (RLS) in HD patients, although the precise association between insomnia and HD remains unclear. One study identified uremia, iron deficiency, and diabetes mellitus (DM) type 2 as potential causes of RLS, ${ }^{18}$ and these conditions are also associated with HD or the state of patients needing HD.

Regarding the associations between insomnia or RLS and characteristics of HD patients, Yildiz et al and Einollahi et al reported that DM was significantly associated with insomnia and RLS and that HD patients with DM were significantly more likely to have poor quality of sleep and RLS. ${ }^{13,15}$ Ezzat and Mohab investigated the association between insomnia and laboratory data and reported that treatment of anemia, hyperphosphatemia, and hypoalbuminemia could improve the insomnia of HD patients. ${ }^{16}$ In addition to these internal medical factors, some studies have investigated the contribution of depression and quality of life (QOL) to insomnia. ${ }^{19-21}$ Trbojevic-Stankovic et al reported that insomnia or poor sleep quality and depression, as evaluated using the Beck Depression Inventory (BDI), were related to each other. ${ }^{19}$ Using the Kidney Disease Quality of Life questionnaire, Parvan et al studied QOL in HD patients and showed that lower QOL was associated with poor sleep quality. ${ }^{20}$ Afsar and Elsurer studied both depression and QOL in HD patients using the BDI and the 36-item Short Form Health Survey (SF-36) and showed that the BDI score and SF-36 score were significantly positively and negatively associated with insomnia evaluated using the Pittsburgh Sleep Quality Index (PSQI) and the Epworth Sleepiness Scale questionnaire, respectively. ${ }^{21}$

Thus, many studies have investigated insomnia in HD patients and the factors responsible for this association, although the influence of the duration of HD has not been thoroughly investigated. As the result of daily bed rest or other influences from long-term HD, a longer HD duration might influence insomnia in HD patients. In the present study, we investigated the factors, especially the duration of HD, associated with insomnia in HD patients.

\section{Methods}

\section{Participants}

This study was conducted between April and June 2015. A total of 138 patients (96 males and 42 females) undergoing HD were recruited from Oyokyo Kidney Research Institute in Japan. Clinical information was obtained from medical records. Blood sampling was performed no later than 2 weeks prior to assessment. Albumin, total protein, sodium, potassium, uric acid, creatinine, blood urea nitrogen, and the amount of HD were measured with standard analytical techniques.
This study was approved by the Ethics Committee of the Hirosaki University School of Medicine, and all subjects provided written informed consent before participating in this study.

\section{Assessment of insomnia, RLS, depression, and health-related QOL}

We used the Japanese version of the PSQI to assess the quality of sleep of the subjects in the present study. ${ }^{22.23}$ The PSQI consists of seven components: subjective sleep quality, sleep latency, sleep duration, habitual sleep efficiency, sleep disturbances, use of sleep medication, and daytime dysfunction. Each component (C), C1-C7, is scored from 0 to 3, and the maximum total PSQI score is 21 . Subjects with total PSQI score of $\geq 5$ show poor sleep quality, ${ }^{24}$ thus, in the present study, we defined those subjects with poor sleep quality as "insomnia subjects".

We recorded the symptoms of RLS according to the criteria put forth by the International Restless Legs Syndrome Study Group. ${ }^{25}$ The diagnostic criteria for RLS include the following: an urge to move the legs with or without abnormal sensations, worsening of symptoms at rest, improvement in symptoms with activity, and worsening of symptoms in the evening/night.

The Japanese version of the Center for Epidemiologic Studies Depression Scale (CES-D) was also administered to all participants to measure their depressive status. ${ }^{26}$ This questionnaire has been widely used to measure depressive symptoms in community populations, and it is also used as a screening tool for depression. ${ }^{27}$ The CES-D is a 20 -item self-report scale that focuses on depressive symptoms within the week prior to administration of the questionnaire. The maximum score is set at 60 , and higher scores are associated with depression. CES-D scores of $\geq 16$ have generally been thought to indicate clinically relevant depressive symptoms, including both minor or subthreshold depression and major depressive disorder. ${ }^{28,29}$ Participants whose CES-D score was $\geq 16$ were defined as having CES-D depression.

Health-related QOL of the subjects was assessed using the Short Form 8 Health Survey (SF-8). This survey includes eight items, and each item corresponds to an item of the SF-36; the SF-8 is shorter and easier to use than the SF-36. ${ }^{30}$ In the present study, we used a Japanese version of the SF-36, which was translated and validated by Fukuhara and Suzukamo. ${ }^{31}$

\section{Statistical analysis}

We used $t$-tests and chi-square tests to compare the subjects with and without insomnia with regard to demographic and 
clinical data. Additionally, we compared the demographic and clinical data of the subjects by assigning them to two groups according to the median HD duration: a short HD duration group and a long HD duration group.

We performed multiple regression analysis using the total PSQI score as the dependent variable for subjects with a short HD duration and using demographic data, the SF-8 score, depression, and RLS as dependent variables for those with long HD duration.

The data were analyzed using SPSS for Windows 22 (IBM Corporation, Armonk, NY, USA). A $P$-value $\leq 0.05$ was considered statistically significant.

\section{Results}

\section{Demographic and clinical data of the entire group and comparisons between subjects with and without insomnia}

Table 1 shows the demographic and clinical data of all the subjects. The mean age, HD time, HD duration, SF-8 score, CES-D score, and total PSQI score of the subjects were $66.8 \pm 11.9$ years, $3.8 \pm 0.4$ hours, $6.5 \pm 6.2$ years, $15.8 \pm 6.2$, $8.2 \pm 7.2$, and $4.9 \pm 3.0$, respectively. The median duration of HD was 4 years.

According to the definitions described in the "Assessment of insomnia, RLS, depression, and health-related QOL" section, 21 subjects $(15.2 \%)$ had depression, $26(18.8 \%)$ had RLS, and 75 (54.3\%) experienced poor sleep quality or had insomnia.

Additionally, Table 1 shows the results of $t$-tests comparing the variables of subjects with and without insomnia. The normal group included 63 subjects, and the insomnia group included 75 subjects. Total PSQI score and scores of $\mathrm{C} 2$, C3, C5, C6, and C7 showed significant differences between the two groups, although scores of $\mathrm{C} 1$ and $\mathrm{C} 4$ did not show significant differences.

Similarly, the SF-8 score and CES-D score showed significant differences between the two groups; the insomnia group showed significantly higher SF-8 and CES-D scores than the normal group. There were no significant differences in demographic variables or other clinical variables.

\section{Comparison between subjects with a short and a long HD duration}

Table 2 shows the results of $t$-tests comparing the variables of the short HD duration group and long HD duration group. The subjects in the short HD duration group had an HD duration $\leq 4$ years. The subjects in the long HD duration group had an HD duration $\geq 5$ years. The short HD duration group included 72 subjects, and the long HD duration group included 66 subjects.

Table I Demographic and clinical data of all the subjects and comparison of subjects with and without insomnia

\begin{tabular}{|c|c|c|c|c|}
\hline Variables & Total $(n=138)$ & Normal $(n=63)$ & Insomnia $(n=75)$ & $P$-value \\
\hline Age (years) & $66.8 \pm 11.9$ & $67.4 \pm 12.5$ & $66.4 \pm 11.5$ & 0.608 \\
\hline Sex (male:female) & $96: 42$ & $46: 17$ & $50: 25$ & $0.419^{a}$ \\
\hline HD time (hours) & $3.8 \pm 0.4$ & $3.8 \pm 0.4$ & $3.8 \pm 0.4$ & 0.955 \\
\hline HD duration (years) & $6.5 \pm 6.2$ & $6.0 \pm 6.3$ & $6.9 \pm 6.1$ & 0.413 \\
\hline BUN (mg/dL) & $64.6 \pm 16.9$ & $65.8 \pm 16.7$ & $63.6 \pm 17.1$ & 0.466 \\
\hline Cre (mg/dL) & $10.4 \pm 3.1$ & $10.4 \pm 3.2$ & $10.4 \pm 3.0$ & 0.934 \\
\hline SF-8 & $15.8 \pm 6.2$ & $14.2 \pm 4.6$ & $17.2 \pm 7.0$ & $0.003^{*}$ \\
\hline CES-D & $8.2 \pm 7.2$ & $6.0 \pm 6.5$ & $9.9 \pm 7.4$ & $0.002 *$ \\
\hline Depression, n (\%) & $2 \mathrm{I}(\mathrm{I} .2 \%)$ & 7 (II.I\%) & 14 (I8.7\%) & $0.218^{\mathrm{a}}$ \\
\hline RLS, n (\%) & $26(18.8 \%)$ & I5 (23.8\%) & II (I4.7\%) & $0.171^{\mathrm{a}}$ \\
\hline \multicolumn{5}{|l|}{ PSQI } \\
\hline $\mathrm{Cl}$ - quality & $0.8 \pm 0.9$ & $0.3 \pm 0.6$ & $1.3 \pm 0.9$ & 0.195 \\
\hline C2 - latency & $1.5 \pm 1.2$ & $0.7 \pm 0.9$ & $2.1 \pm 1.0$ & $0.000^{*}$ \\
\hline C3 - duration & $0.4 \pm 0.8$ & $0.1 \pm 0.4$ & $0.6 \pm 0.9$ & $0.000 *$ \\
\hline C4 - efficacy & $0.0 \pm 0.3$ & $0.0 \pm 0.1$ & $0.1 \pm 0.4$ & 0.279 \\
\hline C5 - disturbances & $0.7 \pm 0.5$ & $0.6 \pm 0.5$ & $0.9 \pm 0.4$ & $0.00 I^{*}$ \\
\hline C6-medication & $1.0 \pm 1.4$ & $0.4 \pm 1.0$ & $1.5 \pm 1.5$ & $0.000 *$ \\
\hline C7 - daytime dysfunction & $0.5 \pm 0.8$ & $0.2 \pm 0.5$ & $0.7 \pm 0.9$ & $0.000 *$ \\
\hline PSQI total score & $4.9 \pm 3.0$ & $2.4 \pm 1.4$ & $7.0 \pm 2.2$ & $0.000 *$ \\
\hline Insomnia, n (\%) & 75 (54.3\%) & - & - & - \\
\hline
\end{tabular}

Notes: Data presented as mean \pm standard deviation unless stated otherwise. “-” indicates not applicable. " Chi-square test. $* P<0.01$.

Abbreviations: HD, hemodialysis; BUN, blood urea nitrogen; Cre, creatinine; SF-8, Short Form 8 Health Survey; CES-D, Center for Epidemiologic Studies Depression Scale; RLS, restless legs syndrome; PSQI, Pittsburgh Sleep Quality Index. 
Table 2 Comparison of subjects with a short and a long HD duration

\begin{tabular}{llll}
\hline Variables & Short HD duration $(\mathbf{n}=\mathbf{7 2})$ & Long HD duration $(\mathbf{n}=\mathbf{6 6})$ & P-value \\
\hline Age (years) & $68.9 \pm 11.3$ & $64.6 \pm 12.3$ & $0.032^{*}$ \\
Sex (male:female) & $49: 23$ & $47: 19$ & $0.715^{\mathrm{a}}$ \\
HD time (hours) & $3.7 \pm 0.5$ & $3.9 \pm 0.3$ & $0.004^{* *}$ \\
HD duration (years) & $1.8 \pm 1.4$ & $11.5 \pm 5.4$ & $0.000^{* *}$ \\
BUN (mg/dL) & $65.0 \pm 16.2$ & $64.2 \pm 17.8$ & 0.797 \\
Cre (mg/dL) & $9.6 \pm 2.8$ & $11.2 \pm 3.1$ & $0.002^{* *}$ \\
SF-8 & $15.7 \pm 5.9$ & $16.0 \pm 6.6$ & 0.775 \\
CES-D & $7.5 \pm 7.1$ & $8.9 \pm 7.4$ & 0.241 \\
Depression, n (\%) & $9(12.5 \%)$ & $12(18.2 \%)$ & $0.477^{\mathrm{a}}$ \\
RLS, n (\%) & $13(18.1 \%)$ & $13(19.7 \%)$ & $0.805^{\mathrm{a}}$ \\
PSQI & & & 0.382 \\
CI - quality & $0.9 \pm 1.0$ & $0.8 \pm 0.7$ & 0.309 \\
C2 - latency & $1.4 \pm 1.2$ & $1.6 \pm 1.1$ & 0.715 \\
C3 - duration & $0.4 \pm 0.8$ & $0.4 \pm 0.8$ & 0.512 \\
C4 - efficacy & $0.0 \pm 0.2$ & $0.1 \pm 0.4$ & 0.192 \\
C5 - disturbances & $0.8 \pm 0.5$ & $0.7 \pm 0.5$ & 0.130 \\
C6 - medication & $0.8 \pm 1.3$ & $1.2 \pm 1.5$ & $0.02 I^{*}$ \\
C7 - daytime dysfunction & $0.4 \pm 0.6$ & $0.7 \pm 0.9$ & 0.179 \\
PSQI total score & $4.6 \pm 3.0$ & $5.3 \pm 2.8$ & $0.174^{\mathrm{a}}$ \\
Insomnia, n (\%) & $35(48.6 \%)$ & $40(60.6 \%)$ & \\
\hline Notes Dat pres &
\end{tabular}

Notes: Data presented as mean \pm standard deviation unless stated otherwise. ${ }^{a}$ Chi-square test. $* P<0.05$. $* * P<0.0$ I.

Abbreviations: HD, hemodialysis; BUN, blood urea nitrogen; Cre, creatinine; SF-8, Short Form 8 Health Survey; CES-D, Center for Epidemiologic Studies Depression Scale; RLS, restless legs syndrome; PSQI, Pittsburgh Sleep Quality Index.

Age was significantly higher in the short HD duration group than the long HD duration group. HD time, HD duration, and creatinine levels were significantly higher in the long HD duration group than in the short HD duration group.

There was no significant difference in the prevalence rate of insomnia between the two groups. There was also no significant difference in the total PSQI score or the scores of individual components except $\mathrm{C} 7$ (daytime dysfunction); the score of $\mathrm{C} 7$ was significantly higher in the long HD duration group than the short HD duration group.

\section{Multiple regression analysis of the total PSQI score in subjects with a short and a long HD duration}

Table 3 shows the results of multiple regression analysis of the total PSQI score in subjects in short HD duration group and long HD duration group using demographic data, the SF-8 score, depression, and RLS as dependent variables.

In the short HD duration group, no variables showed significant associations. In the long HD duration group, age and SF-8 score showed significant associations; age was negatively significantly associated with the total PSQI score, and the SF-8 score was positively significantly associated with the total PSQI score. Additionally, only the correlation between PSQI score and SF-8 found by single correlation analysis was significant.

\section{Discussion}

In the present study, we investigated the factors associated with insomnia in HD patients, with a particular focus on the HD duration of the patients. Our results showed a higher prevalence of insomnia in HD patients. There was no significant difference between the prevalence rate of insomnia in the short and long HD duration groups, while the score

Table 3 Multiple regression analysis of the total PSQI score for subjects with a short and a long HD duration

\begin{tabular}{llll}
\hline Groups & Beta coefficient & t-value & $P$-value \\
\hline Short HD duration & & & \\
Age (years) & -0.170 & -1.280 & 0.205 \\
Sex & 0.022 & 0.177 & 0.860 \\
HD time (hours) & -0.042 & -0.286 & 0.776 \\
HD duration (years) & -0.072 & -0.512 & 0.610 \\
SF-8 & 0.191 & 1.354 & 0.180 \\
Depression & -0.051 & -0.358 & 0.722 \\
RLS & 0.096 & 0.773 & 0.442 \\
Long HD duration & & & \\
Age (years) & -0.281 & -2.104 & $0.040^{*}$ \\
Sex & 0.112 & 0.895 & 0.374 \\
HD time (hours) & 0.025 & 0.208 & 0.836 \\
HD duration (years) & -0.126 & -0.976 & 0.333 \\
SF-8 & 0.348 & 2.462 & $0.017^{*}$ \\
Depression & 0.078 & 0.548 & 0.586 \\
RLS & -0.183 & -1.432 & 0.157 \\
\hline
\end{tabular}

Note: $* P<0.05$.

Abbreviations: PSQI, Pittsburgh Sleep Quality Index; HD, hemodialysis; SF-8, Short Form 8 Health Survey; RLS, restless legs syndrome. 
of C7 (daytime dysfunction) of the PSQI was significantly higher and the SF-8 score showed a significant association with the PSQI score in the long HD duration group. Thus, in patients with a longer HD duration, insomnia might be closely associated with QOL. This is the first report to investigate insomnia in HD patients by classifying them according to their HD duration.

With the exception of $\mathrm{C} 1$ "quality" and C4 "efficacy", there were significant differences between the PSQI component scores of the normal and insomnia groups. $\mathrm{C} 1$ is a subjective assessment by patients about their quality of sleep, while $\mathrm{C} 4$ assesses the percentage of total number of hours asleep during the total number of hours in bed. Patients diagnosed without insomnia might think that their sleep was poor or short considering their hours in bed. There has been no report discussing the differences in PSQI component scores of HD patients with and without insomnia evaluated by the PSQI, although patients might be equally influenced by their time in bed during HD and think their sleep to be poor.

We showed that the prevalence rate of insomnia in all HD patients was $54.3 \%$. The reported range of prevalence rates of insomnia in HD patients is relatively wide. Ezzat and Mohab reported that the prevalence rate of insomnia in HD patients was $54 \%$, a value similar to the $54.3 \%$ we have shown in the present study. ${ }^{16}$ On the other hand, several studies of HD patients evaluated insomnia with the same method used in the present study, that is, a total PSQI score of $\geq 5$, and reported prevalence rates somewhat different from the value shown in the present study; Parvan et al and Iliescu et al reported rates of $83.3 \%$ and $71 \%$, respectively. ${ }^{20,32}$ Although we used the same method to assess insomnia, differences in characteristics of the backgrounds of the patients from each hospital or the system of the hospitals might have influenced the prevalence rate. For example, more patients might have been taking hypnotics or psychotropic drugs to obtain better sleep in some hospitals, and psychiatrists or experts in insomnia treatment might have participated in sleep studies in some hospitals.

We hypothesized that the long HD duration group would show a higher prevalence of insomnia because we thought that the sleep-wake rhythm of patients might change with a longer HD duration, but there was no significant difference in the prevalence rate of insomnia between the short and long HD duration groups. The psychological reaction to HD or the introduction of HD and the practice of HD in the long HD duration group might have offset each other. In the present study, the patients were divided into two groups according to the median HD duration. Further studies using other methods to classify patients by HD duration might obtain additional information about potential differences in the prevalence rate of insomnia in relation to HD duration.

Of the PSQI component scores, only C7 showed a significant difference between the short and long HD duration groups. C7 indicates the "daytime dysfunction" of the patient, and the questionnaire asked whether the patient had trouble staying awake during daytime activity and keeping up enthusiasm to get things done. In other words, $\mathrm{C} 7$ shows the influence of insomnia on whether the patient performed daytime activities without trouble, indicating that patients with higher scores on $\mathrm{C} 7 \mathrm{might}$ have a poorer QOL. This finding may also relate to the association between PSQI score and SF-8 score in the long HD duration group, according to the multiple regression analysis shown in Table 3 and the single correlation analysis. Particularly for patients with a longer HD duration, treatment for insomnia and psycho-education about better sleep habits and staying awake during daytime might improve the QOL.

There were some limitations to the present study. First, we did not investigate the medication of the patients; we only asked how they took medicine to help their sleep (as one question of the PSQI) but did not investigate the type or amount of medication. Indeed, we might be able to determine better prescriptions for $\mathrm{HD}$ patients to improve their insomnia or poor QOL associated with insomnia by investigating their medication history. Second, we did not assess comorbid diseases of the HD patients and did not investigate the method used for HD. Previous studies have reported that DM might be associated with a higher prevalence of insomnia or RLS in HD patients. ${ }^{13,15}$ The sleep-associated activities performed by patients during HD might be influenced by the method used for HD. Thus, treatments for insomnia should likely be tailored according to comorbid diseases and the HD method. Third, we investigated only subjects who were hemodialyzed during an afternoon session. The subjects in another session might experience a suitable QOL during their HD session and might show different characteristics from those observed in the present study.

\section{Conclusion}

We investigated the differences in the characteristics of HD patients with a short or long HD duration, and the results suggest that HD patients with a long HD duration might experience an influence of insomnia on their daily activities and a potential significant association between insomnia and QOL. Therefore, we should pay greater attention to poor QOL and trouble in daily activities caused by insomnia in patients with a longer duration of HD. 


\section{Acknowledgments}

The authors would like to thank all of their coworkers of this study for their skillful contributions to collecting and managing the data.

\section{Disclosure}

The authors report no conflicts of interest in this work.

\section{References}

1. Kimmel PL, Weihs K, Peterson RA. Survival in hemodialysis patients: the role of depression. J Am Soc Nephrol. 1993;4(1):12-27.

2. Finkelstein FO, Finkelstein SH. Depression in chronic dialysis patients: assessment and treatment. Nephrol Dial Transplant. 2000;15(12): 1911-1913.

3. Kimmel PL, Peterson RA, Weihs KL, et al. Multiple measurements of depression predict mortality in a longitudinal study of chronic hemodialysis outpatients. Kidney Int. 2000;57(5):2093-2098.

4. Kimmel PL. Psychosocial factors in dialysis patients. Kidney Int. 2001; 59(4):1599-1613.

5. Lopes AA, Bragg J, Young E, et al. Depression as a predictor of mortality and hospitalization among hemodialysis patients in the United States and Europe. Kidney Int. 2002;62(1):199-207.

6. Mitrou GI, Grigoriou SS, Konstantopoulou E, et al. Exercise training and depression in ESRD: a review. Semin Dial. 2013;26(5):604-613.

7. Chilcot J, Spencer BW, Maple H, Mamode N. Depression and kidney transplantation. Transplantation. 2014;97(7):717-721.

8. Zalai D, Szeifert L, Novak M. Psychological distress and depression in patients with chronic kidney disease. Semin Dial. 2012;25(4): $428-438$.

9. Watnick S, Wang PL, Demadura T, Ganzini L. Validation of 2 depression screening tools in dialysis patients. Am J Kidney Dis. 2005; 46(5):919-924.

10. Hedayati SS, Bosworth HB, Kuchibhatla M, Kimmel PL, Szczech LA. The predictive value of self-report scales compared with physician diagnosis of depression in hemodialysis patients. Kidney Int. 2006;69(9): $1662-1668$.

11. Cukor D, Coplan J, Brown C, Peterson RA, Kimmel PL. Course of depression and anxiety diagnosis in patients treated with hemodialysis: a 16-month follow-up. Clin J Am Soc Nephrol. 2008;3(6):1752-1758.

12. Lopes AA, Albert JM, Young EW, et al. Screening for depression in hemodialysis patients: associations with diagnosis, treatment, and outcomes in the DOPPS. Kidney Int. 2004;66(5):2047-2053.

13. Yildiz D, Kahvecioglu S, Buyukkoyuncu N, et al. Restless-legs syndrome and insomnia in hemodialysis patients. Ren Fail. 2016;38(2): 194-197.

14. Yazdi Z, Sadeghniiat-Haghighi K, Kazemifar AM, Kordi A, Naghipour S. Restless leg syndrome in hemodialysis patients: a disorder that should be noticed. Saudi J Kidney Dis Transplant. 2015;26(3):625-650.

15. Einollahi B, Motalebi M, Rostami Z, Nemati E, Salesi M. Sleep quality among Iranian hemodialysis patients: a multicenter study. Nephrourol Mon. 2015;7(1):e23849.
16. Ezzat H, Mohab A. Prevalence of sleep disorders among ESRD patients. Ren Fail. 2015;37(6):1013-1039.

17. Chavoshi F, Einollahi B, Sadeghniat Haghighi K, Saraei M, Izadianmehr N. Prevalence and sleep related disorders of restless leg syndrome in hemodialysis patients. Nephrourol Mon. 2015;7(2):e24611.

18. Allen RP. Controversies and challenges in defining the etiology and pathophysiology of restless legs syndrome. Am J Med. 2007; 120(1 Suppl 1):S13-S21.

19. Trbojevic-Stankovic J, Stojimirovic B, Bukumiric Z, et al. Depression and quality of sleep in maintenance hemodialysis patients. Srp Arh Celok Lek. 2014;142(7-8):437-443.

20. Parvan K, Lakdizaji S, Roshangar F, Mostofi M. Quality of sleep and its relationship to quality of life in hemodialysis patients. J Caring Sci. 2013;2(4):295-304.

21. Afsar B, Elsurer R. The relationship between sleep quality and daytime sleepiness and various anthropometric parameters in stable patients undergoing hemodialysis. J Ren Nutr. 2013;23(4):296-301.

22. Doi Y, Minowa M, Uchiyama M, Okawa M. [Development of the Japanese version of the Pittsburgh Sleep Quality Index]. Jpn J Psychiatr Treat. 1998;13:755-763. Japanese.

23. Buysse DJ, Reynolds CF 3rd, Monk TH, Berman SR, Kupfer DJ. The Pittsburgh Sleep Quality Index: a new instrument for psychiatric practice and research. Psychiatry Res. 1989;28(2):193-213.

24. Buysse DJ, Hall ML, Strollo PJ, et al. Relationships between the Pittsburgh Sleep Quality Index (PSQI), Epworth Sleepiness Scale (ESS), and clinical/polysomnographic measures in a community sample. J Clin Sleep Med. 2008;4(6):563-571.

25. Allen RP, Picchietti D, Hening WA, Trenkwalder C, Walters AS, Montplaisi J. Restless legs syndrome: diagnostic criteria, special considerations, and epidemiology. A report from the restless legs syndrome diagnosis and epidemiology workshop at the National Institutes of Health. Sleep Med. 2003;4(2):101-119.

26. Shima S, Shikano T, Kitamura T. [New self-rating scales for depression]. Clin Psychiatry. 1985;27:717-723. Japanese.

27. Zich JM, Attkisson CC, Greenfield TK. Screening for depression in primary care clinics: the CES-D and the BDI. Int $J$ Psychiatry Med. 1990;20(2):259-277.

28. Berkman LF, Berkman CS, Kasl S, et al. Depressive symptoms in relation to physical health and functioning in the elderly. Am J Epidemiol. 1986; 124(3):372-388.

29. Beekman AT, Deeg DJ, Van Limbeek J, Braam AW, De Vries MZ, Van Tilburg W. Criterion validity of the Center for Epidemiologic Studies Depression scale (CES-D): results from a community-based sample of older subjects in the Netherlands. Psychol Med. 1997;27(1): 231-235.

30. Ware JE Jr, Sherbourne CD. The MOS 36-item short-form health survey (SF-36). I. Conceptual framework and item selection. Med Care. 1992;30(6):473-483.

31. Fukuhara S, Suzukamo Y. [Manual of the SF-8 Japanese Version]. Kyoto: Institute for Health Outcomes \& Process Evaluation Research; 2004. Japanese.

32. Iliescu EA, Coo H, McMurray MH, et al. Quality of sleep and health-related quality of life in haemodialysis patients. Nephrol Dial Transplant. 2003;18(1):126-132.
Neuropsychiatric Disease and Treatment

\section{Publish your work in this journal}

Neuropsychiatric Disease and Treatment is an international, peerreviewed journal of clinical therapeutics and pharmacology focusing on concise rapid reporting of clinical or pre-clinical studies on a range of neuropsychiatric and neurological disorders. This journal is indexed on PubMed Central, the 'PsycINFO' database and CAS,

\section{Dovepress}

and is the official journal of The International Neuropsychiatric Association (INA). The manuscript management system is completely online and includes a very quick and fair peer-review system, which is all easy to use. Visit http://www.dovepress.com/testimonials.php to read real quotes from published authors. 